18,07

\title{
Влияние азотного допирования и механического напряжения на адсорбционную способность графдиена
}

\author{
(С) И.Ю. Долинский ${ }^{1}$, К.С. Гришаков ${ }^{1,2, \uparrow, ~ В . С . ~ П р у д к о в с к и и ̆ ~}{ }^{2,3}$ \\ ${ }^{1}$ Национальный исследовательский ядерный университет „МИФИ“, \\ Москва, Россия \\ ${ }^{2}$ Научно-исследовательский институт проблем развития научно-образовательного потенциала молодежи, \\ Москва, Россия \\ ${ }^{3}$ University of Crete, \\ Heraklion, Greece \\ ฯ E-mail: ksgrishakov@yahoo.com
}

(Поступила в Редакцию 18 июля 2018 г.)

Проведено квантово-химическое моделирование адсорбции атомарного водорода на чистый и допированный азотом графдиен. Выделены предпочтительные узлы, адсорбция на которые наиболее выгодна энергетически. Показано, что наличие азота приводит к существенному увеличению адсорбционной способности листа. Продемонстрирована способность допированного азотом графдиена обратимо растягиваться на $4 \%$ под действием внешнего механического напряжения. Установлено, что механическое натяжение позволяет управлять адсорбционными свойствами как чистого, так и допированного графдиена.

Работа поддержана РФФИ, проект 16 32-60081 mol_a_dk.

DOI: 10.21883/FTT.2019.02.47144.215

\section{1. Введение}

Атомы углерода могут находиться в состояниях с различными типами гибридизации $\left(s p, s p^{2}, s p^{3}\right)$, благодаря чему существует множество аллотропных модификаций углерода. Самыми распространенными из них являются алмаз $\left(s p^{3}\right)$ и графит $\left(s p^{2}\right)$. В последние десятилетия было синтезировано множество новых аллотропов, в том числе фуллерены, нанотрубки и графен, для которых характерна $s p^{2}$-гибридизация.

Известны также более сложные системы со „смешанными“ типами гибридизации. Примерами таких аллотропов являются графин [1] и графдиен [2], состоящие из атомов углерода, которые находятся в $s p$ - и $s p^{2}$-состояниях. В графдиене, который является объектом исследования в данной работе, атомы углерода образуют гексагональные кольца, которые связаны между собой двумя ацетиленовыми связями.

Пленки графдиена были впервые синтезированы в 2010 г. [3]. В последующих экспериментальных работах удалось получить структуры более высокого качества, а также синтезировать различные морфологии на основе графдиена - графдиеновые нанотрубки [4], нанопровода [5] и наностенки [6].

Успешный синтез графдиена стимулировал множество исследований, направленных на изучение его электронных, структурных, оптических и механических свойств. В отличие от графена графдиен является полупроводником. Теоретические расчеты предсказывают ширину запрещенной зоны в диапазоне $0.46-1.22 \mathrm{eV}$ в зависимости от методики расчета и используемого функционала [7-9]. Графдиен является более мягким материалом по сравнению с графином за счет меньшего числа $\mathrm{C}-\mathrm{C}$-связей [10]. Под действием внешних механических деформаций ширина его запрещенной зоны увеличивается, что позволяет управлять ее величиной [10]. Теоретически рассчитанная подвижность электронов в монослое графдиена может достигать $10^{5} \mathrm{~cm}^{2} \mathrm{~V}^{-1} \mathrm{~s}^{-1}$ при комнатной температуре [11], что близко к соответствующему значению для графена [12]. Однако измеренная подвижность в синтезированных нанокристалах графдиена составляетвсего $7.1 \cdot 10^{2} \mathrm{~cm}^{2} \mathrm{~V}^{-1} \mathrm{~s}^{-1}$ при комнатной температуре [13], что связано с недостаточным качеством используемых образцов. Определенная из эксперимента проводимость многослойных графдиеновых пленок и графдиеновых нанопроводов составляет $2.5 \cdot 10^{-4}$ и $1.9 \cdot 10^{3} \mathrm{~S} / \mathrm{m}[13,14]$, соответственно, то есть принимает значения, типичные для полупроводников. Графдиен проявляет высокую нелинейную оптическую восприимчивость, а также высокую эффективность флуоресценции и термостойкость [15].

Благодаря своим отличительным свойствам, графдиен может найти практическое применение в наноэлектронике [16-18], нанофотонике [18], фотоэлектронике [19] и спинтронике [20]. Его предлагается использовать в качестве полевого транзистора, работающего при комнатной температуре [21]. В отличие от графена, он не требует приложения внешнего электрического поля или структурной модификации для открытия запрещенной зоны. На основе графдиена могут быть созданы солнечные элементы [22-24], литий-ионные аккумуляторы [17,25,26], конденсаторы [25], катализаторы [19,27,28] и молекулярные датчики [29]. Графдиен может использоваться как источник полевой эмиссии [30,31]. 
Как и другие двумерные материалы, графдиен характеризуется очень большой удельной поверхностью. Наличие свободных связей позволяет графдиену присоединять водород [18,32], кислород [27], галогены [18] и другие функциональные группы [33,34]. Адсорбционные свойства графдиена предлагается использовать для хранения водорода [17], создания мембран для очистки водорода $[16,35]$, разделения газов $\mathrm{CO}_{2} / \mathrm{N}_{2} / \mathrm{CH}_{4}$ [36] и фильтрации воды [37].

Множество потенциальных приложений делают актуальным вопрос о возможности управления адсорбционными свойствами графдиена. Одним из известных способов модификации углеродных наноструктур является замещающее азотное допирование - замена одного или нескольких атомов углерода атомами азота [22,28,35]. Выбор именно азота обусловлен тем, что этот элемент легко встраивается в углеродную сеть. Графдиен, допированный азотом, был получен в 2014 г. на поверхности меди с помощью реакции кросс-сочетания с использованием гексаэтинилбензола в качестве прекурcopa $[28,38]$. Азотное допирование многих углеродных структур приводит к увеличению их адсорбционной способности $[39,40]$. Поэтому можно ожидать, что допирование окажет влияние на адсорбционные свойства графдиена. Механическое напряжение листа открывает дополнительные возможности для управления адсорбционной способностью графена [41-43] и может оказаться полезным также и для графдиена.

Настоящая работа посвящена моделированию адсорбции водорода на чистый и допированный азотом графдиен. Цель работы состоит в исследовании влияния механических напряжений и замещающего допирования на адсорбционную способность графдиена.

\section{2. Методика расчета}

Графдиен моделировался при помощи сверхъячейки $\mathrm{C}_{162}$, содержащей $3 \times 3$ элементарных ячеек (см. рис. 1). Для случая $\mathrm{N}$-легированного графдиена использовалась аналогичная сверхьячейка $\mathrm{C}_{161} \mathrm{~N}$, в которой один из атомов углерода замещен атомом азота. В плоскости листа использовались периодические граничные условия, а в перпендикулярном направлении - свободные. Выбранный размер ячейки достаточен для того, чтобы адсорбированный водород и его периодические образы не взаимодействовали друг с другом.

Для описания межатомного взаимодействия использовалась неортогональная модель сильной связи $[44,45]$ с параметризацией [45]. Эта полуэмпирическая модель обладает существенно большей скоростью расчета по сравнению с ab initio подходами, и корректно описывает системы с углеродно-азотным скелетом [46,47], а также широкий класс соединений с различными типами углеродных каркасов - графен [48], фуллерены [49], призманы $[50,51]$ и их производные [52] и системы

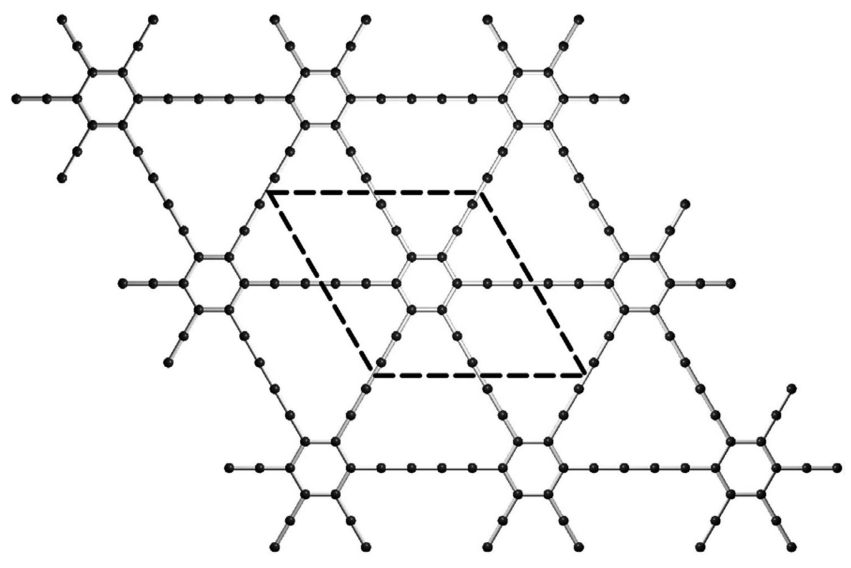

Рис. 1. Сверхъячека графдиена, содержащая 162 атома углерода.

с различными типами гибридизации [53,54]. Геометрическая оптимизация графдиена проводилась методом градиентного спуска: атомы смещались в направлении действующих на них сил, пока эти силы не становились меньше $0.001 \mathrm{eV} / \AA$. Приложенная к структуре деформация растяжения учитывалась посредством соразмерного (на одинаковое количество процентов) увеличения обоих периодов сверхъячейки.

Энергия адсорбции водорода $E_{\text {ads }}$ вычислялась по формуле $E_{\mathrm{ads}}=E\left(\mathrm{~S}_{0}+\mathrm{H}\right)-E\left(\mathrm{~S}_{0}\right)-E(\mathrm{H})$, где $\mathrm{S}_{0}=\mathrm{C}_{161} \mathrm{~N}$ или $\mathrm{C}_{162}$, а $E\left(\mathrm{~S}_{0}+\mathrm{H}\right), E\left(\mathrm{~S}_{0}\right)$ и $E(\mathrm{H})$ - оптимизированные значения энергии для сверхъячейки с адсорбированным водородом, для изолированной сверхъячейки и для водорода, соответственно. Отрицательные значения $E_{\text {ads }}$ показывают, что в процессе адсорбции полная энергия системы понижается.

\section{3. Результаты}

Графдиен обладает высокой симметрией, и в его решетке есть только три неэквивалентных положения атомов углерода. Они обозначены буквами А, В и С на рис. 2. Мы рассчитали относительные энергии сверхъячеек $\mathrm{C}_{161} \mathrm{~N}$, которые составили $0,-0.72$ и $0.32 \mathrm{eV}$ для расположений замещающего атома азота в узлах А, B

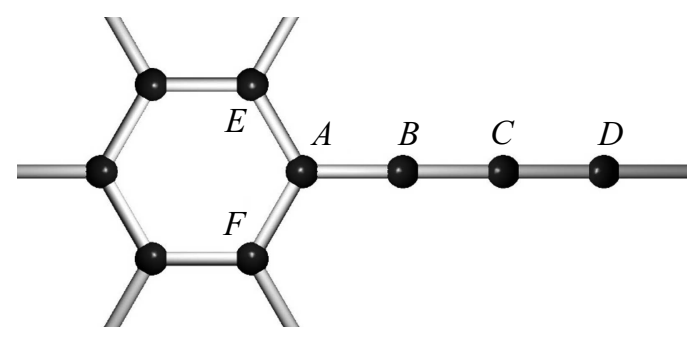

Рис. 2. Обозначения различных атомных позиций в структуре графдиена. 
Энергии адсорбции водорода $(\mathrm{eV})$ на чистый и допированный азотом графдиен в зависимости от расположения замещающего атома азота и адсорбированного атома водорода в решетке графдиена (см. рис. 2)

\begin{tabular}{c|c|c|c|c|c|c|c|c|c|c}
\hline Позиция атома N & \multicolumn{3}{|c|}{$\mathrm{A}$} & \multicolumn{3}{c|}{$\mathrm{B}$} & \multicolumn{3}{c}{$\mathrm{C}$} & \multicolumn{3}{c}{ Нет азота } \\
\hline Позиция атома Н & $\mathrm{B}$ & $\mathrm{E}$ & $\mathrm{F}$ & $\mathrm{A}$ & $\mathrm{C}$ & $\mathrm{B}$ & $\mathrm{D}$ & $\mathrm{A}$ & $\mathrm{B}$ & $\mathrm{C}$ \\
\hline$E_{\mathrm{ads}}, \mathrm{eV}$ & $-{ }^{*}$ & -2.38 & -2.38 & -2.20 & -2.13 & -2.20 & -2.00 & -1.70 & -1.32 & -1.30
\end{tabular}

Примечание. * Происходит разрыв связи $\mathrm{C}-\mathrm{N}$

и С соответственно. Из приведенных данных видно, что наименьшей энергией обладает конфигурация, в которой атом азота находится в положении В. Разница в энергиях сверхъячеек составляет $\sim 1 \mathrm{eV}$, поэтому появление атома азота в положении В значительно более вероятно, в то время как замещение узлов А и С возможно лишь при высоких температурах.

Далее мы рассмотрели адсорбцию одного атома водорода на чистый и допированный азотом графдиен. Рассматривались три возможных расположения замещающего атома азота. Наши расчеты подтвердили, что самая сильная связь между допированным графдиеном и водородом возникает в тех случаях, когда водород адсорбируется на соседний с азотом атом углерода. Повышенная реактивность атомов углерода, находящихся около азота, характерна для углеродных систем и ранее наблюдалась в графене [55] и фуллерене [56]. Полученные энергии адсорбции приведены в таблице. Для чистого графдиена абсолютное значение $E_{\text {ads }}$ максимально при адсорбции водорода на узел А. Наибольший выигрыш в энергии для N-легированного графдиена достигается в случае, когда атом азота находится в узле А, а атом водорода адсорбируется на узел Е или F. Отметим, что наибольшее абсолютное значение $E_{\text {ads }}$ для чистого графдиена превышают соответствующие значения для графена на $\sim 0.2 \mathrm{eV}[55]$, то есть графдиен более реактивен по отношению к водороду. Это объясняется наличием у графдиена дополнительных нескомпенсированных связей. Однако в отличие от графена, в графдиене существуют предпочтительные узлы адсорбции.

Допирование графдиена азотом повышает энергетическую выгоду от адсорбции водорода на узлы, соседние с атомом азота. При этом энергия адсорбции достигает значений, близких к энергии диссоциации молекулы $\mathrm{H}_{2}$ (2.24 eV/atom).

Далее мы изучили влияние растяжения графдиена на энергию адсорбции водорода. Рассматривался как недопированный, так и допированный азотом графдиен. В случае недопированного графдиена, водород находился на самом энергетически выгодном узле (А, см. рис. 2). В случае допированного графдиена, атом азота находился в самом энергетически выгодном положении В, а атом водорода адсорбировался на узел А. Зависимость энергии адсорбции от растяжения графдиена представлена на рисунке 3. Из этого рисунка видно, что при растяжении листа в пределах 4\% связь графдиена с атомом водорода становится все более и более прочной. При растяжении

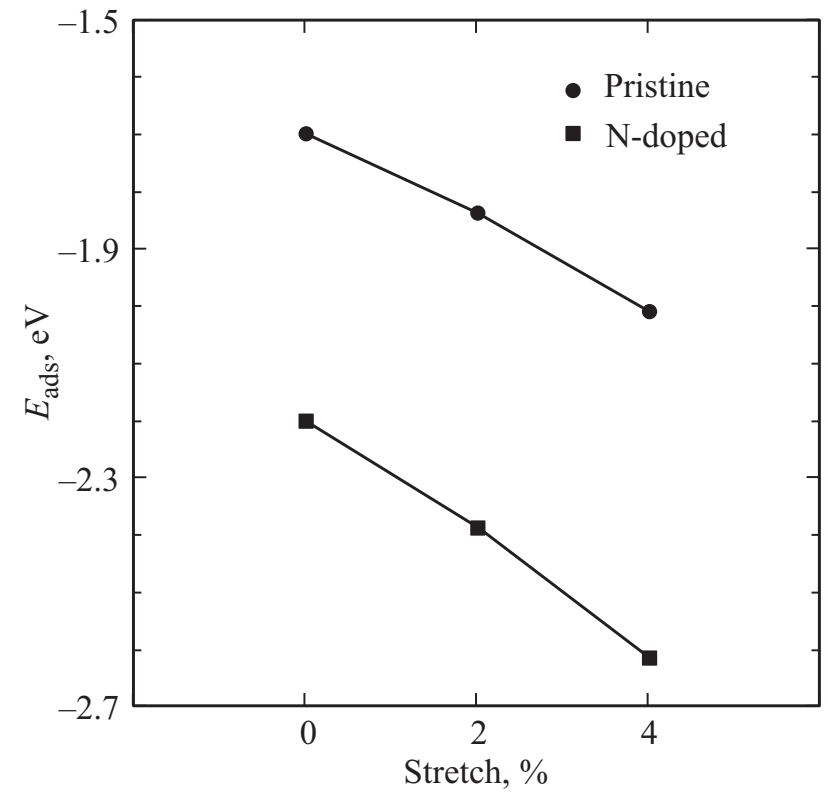

Рис. 3. График зависимости энергии адсорбции водорода на чистый (кружки) и $\mathrm{N}$-легированный (квадраты) графдиен от величины растяжения.

графдиена на 5\% и более мы наблюдали необратимый разрыв $\mathrm{C}-\mathrm{C}$ или $\mathrm{C}-\mathrm{N}-$ связей. Тем не менее, даже небольшого растяжения в пределах 4\% достаточно для существенного варьирования величины $E_{\text {ads }}$.

\section{4. Заключение}

В настоящей работе мы исследовали адсорбцию водорода на растянутый графдиен, допированный атомами азота. Расчеты показали, что растяжение способствует адсорбции как на допированный, так и на недопированный графдиен. Даже небольшого растяжения 4\% достаточно для того, чтобы изменить энергию адсорбции на несколько десятых электрон-вольта. Адсорбция на наиболее активные узлы допированного графдиена приводит к значительно большему энергетическому выигрышу по сравнению с недопированным графдиеном. Этот выигрыш сравним с энергией диссоциации молекулы $\mathrm{H}_{2}$.

Во взаимодействии атома водорода с листом графдиена проявляются те же общие тенденции, что и при его взаимодействии с графеном: допирование и растяжение заметно увеличивают энергию химической 
связи. Графдиен связывается с водородом несколько сильнее графена, однако его низкая прочность ограничивает эффективность управления адсорбцией при помощи механического натяжения. В целом, нам не удалось выявить значительных преимуществ адсорбционных характеристик графдиена по сравнению с графеном. Тем не менее, даже малое механическое натяжение графдиена обеспечивает достаточное изменение энергии адсорбции. При различных натяжениях листа реакция расщепления молекулы $\mathrm{H}_{2}$ с последующим осаждением атомов водорода на графен может быть как эндо-, так и экзотермической. Это открывает принципиальную возможность управлять процессами адсорбции/десорбции водорода на графдиен.

\section{Список литературы}

[1] R. H. Baughman, H. Eckhardt, M. Kertesz. J. Chem. Phys. 87, 6687 (1987).

[2] M.M. Haley, S.C. Brand, J.J. Pak. Angew. Chem. Int. Ed. Eng. 36, 835 (1997).

[3] G. Li, Y. Li, H. Liu, Y. Guo, Y. Li, D. Zhu. Chem. Commun. 46, 3256 (2010).

[4] G. Li, Y. Li, X. Qian, H. Liu, H. Lin, N. Chen, Y. Li. J. Phys. Chem. C 115, 2611 (2011).

[5] X. Qian, Z. Ning, Y. Li, H. Liu, C. Ouyang, Q. Chen, Y. Li. Dalton Trans. 41, 730 (2012).

[6] J. Zhou, X. Gao, R. Liu, Z. Xie, J. Yang, S. Zhang, G. Zhang, H. Liu, Y. Li, J. Zhang, Z. Liu. J. Am. Chem. Soc. 137, 7596 (2015).

[7] N. Narita, S. Nagai, S. Suzuki, K. Nakao. Phys. Rev. B 58, 11009 (1998).

[8] A.L. Ivanovskii. Prog. Solid State Chem. 41, 1 (2013).

[9] G. Luo, X. Qian, H. Liu, R. Qin, J. Zhou, L. Li, Z. Gao, E. Wang, W.-N. Mei, J. Lu, Y. Li, S. Nagase. Phys. Rev. B 84, (2011).

[10] Y. Pei. Physica B 407, 4436 (2012).

[11] M. Long, L. Tang, D. Wang, Y. Li, Z. Shuai. ACS Nano 5, 2593 (2011).

[12] K.I. Bolotin, K.J. Sikes, J. Hone, H.L. Stormer, P. Kim. Phys. Rev. Lett. 101 (2008).

[13] X. Qian, Z. Ning, Y. Li, H. Liu, C. Ouyang, Q. Chen, Y. Li. Dalton Trans. 41, 730 (2012).

[14] G. Li, Y. Li, H. Liu, Y. Guo, Y. Li, D. Zhu. Chem. Commun. 46, 3256 (2010).

[15] J.A. Marsden, M.M. Haley. J. Organic Chem. 70, 10213 (2005).

[16] Y. Jiao, A. Du, M. Hankel, Z. Zhu, V. Rudolph, S.C. Smith. Chem. Commun. 47, 11843 (2011).

[17] K. Srinivasu, S.K. Ghosh. J. Phys. Chem. C 116, 5951 (2012).

[18] J. Koo, M. Park, S. Hwang, B. Huang, B. Jang, Y. Kwon, H. Lee. Phys. Chem. Chem. Phys. 16, 8935 (2014).

[19] Y. Li, L. Xu, H. Liu, Y. Li. Chem. Soc. Rev. 43, 2572 (2014).

[20] X. Chen, P. Gao, L. Guo, Y. Wen, Y. Zhang, S. Zhang. J. Phys. Chem. Solids 105, 61 (2017).

[21] Y. Pan, Y. Wang, L. Wang, H. Zhong, R. Quhe, Z. Ni, M. Ye, W.-N. Mei, J. Shi, W. Guo, J. Yang, J. Lu. Nanoscale 7, 2116 (2015).

[22] J. Li, T. Jiu, C. Duan, Y. Wang, H. Zhang, H. Jian, Y. Zhao, N. Wang, C. Huang, Y. Li. Nano Energy 46, 331 (2018).
[23] H. Du, Z. Deng, Z. Lü, Y. Yin, L. Yu, H. Wu, Z. Chen, Y. Zou, Y. Wang, H. Liu, Y. Li. Synthetic Met. 161, 2055 (2011).

[24] C. Kuang, G. Tang, T. Jiu, H. Yang, H. Liu, B. Li, W. Luo, X. Li, W. Zhang, F. Lu, J. Fang, Y. Li. Nano Lett. 15, 2756 (2015).

[25] K. Wang, N. Wang, J. He, Z. Yang, X. Shen, C. Huang. Electrochim. Acta 253, 506 (2017).

[26] H. Zhang, Y. Xia, H. Bu, X. Wang, M. Zhang, Y. Luo, M. Zhao. J. Appl. Phys. 113, 44309 (2013).

[27] H. Qi, P. Yu, Y. Wang, G. Han, H. Liu, Y. Yi, Y. Li, L. Mao. J. Am. Chem. Soc. 137, 5260 (2015).

[28] R. Liu, H. Liu, Y. Li, Y. Yi, X. Shang, S. Zhang, X. Yu, S. Zhang, H. Cao, G. Zhang. Nanoscale 6, 11336 (2014).

[29] X. Chen, P. Gao, L. Guo, S. Zhang. Sci. Rep. 5, (2015).

[30] G. Li, Y. Li, X. Qian, H. Liu, H. Lin, N. Chen, Y. Li. J. Phys. Chem. C 115, 2611 (2011).

[31] J. Gong, Y. Tang, P. Yang. J. Mol. Structure 1064, 32 (2014).

[32] H. Qiul, X. Sheng. Phys. Lett. A 382, 662 (2018).

[33] N. Ketabi, T.M. Tolhurst, B. Leedahl, H. Liu, Y. Li, A. Moewes. Carbon 123, 1 (2017).

[34] P. Zhang, S. Ma, L.Z. Sun. Appl. Surf. Sci. 361, 206 (2016).

[35] Y. Jiao, A. Du, S.C. Smith, Z. Zhu, S.Z. Qiao. J. Mater. Chem. A 3, 6767 (2015).

[36] L. Zhao, P. Sang, S. Guo, X. Liu, J. Li, H. Zhu, W. Guo. Appl. Surf. Sci. 405, 455 (2017).

[37] M. Bartolomei, E. Carmona-Novillo, M.I. Hernández, J. Campos-Martínez, F. Pirani, G. Giorgi, K. Yamashita. J. Phys. Chem. Lett. 5, 751 (2014).

[38] H. Shang, Z. Zuo, H. Zheng, K. Li, Z. Tu, Y. Yi, H. Liu, Y. Li, Y. Li. Nano Energy 44, 144 (2018).

[39] Z. Zhang, K. Cho. Phys. Rev. B: Condens. Matter Mater. Phys. 75, 075420 (2007).

[40] K.Y. Kang, B.I. Lee, J.S. Lee. Carbon 47, 1171 (2009).

[41] D.W. Boukhvalov, Y.-W. Son. Chem. Phys. Chem. 13, 1463 (2012).

[42] K.P. Katin, V.S. Prudkovskiy, M.M. Maslov. Phys. Lett. A 381, 2686 (2017).

[43] S.Yu. Davydov. Phys. Solid State 59, 845 (2017).

[44] К.П. Катин, М.М. Маслов. Химическая физика 30, 41 (2011).

[45] M.M. Maslov, A.I. Podlivaev, K.P. Katin. Mol. Simulation 42, 305 (2015).

[46] K.P. Katin, M.M. Maslov. J. Phys. Chem. Solids 108, 82 (2017).

[47] Н.Н. Дегтяренко, К.П. Катин, М.М. Маслов. ФТТ 56, 1415 (2014).

[48] Л.А. Опенов, А.И. Подливаев. ФТТ 58, 821 (2016).

[49] А.И. Подливаев, К.П. Катин. Письма в ЖЭТФ 92, 54 (2010).

[50] S.A. Shostachenko, M.M. Maslov, V.S. Prudkovskii, K.P. Katin. Phys. Solid State 57, 1023 (2015).

[51] K.P. Katin, V.S. Prudkovskiy, M.M. Maslov. Physica E 81, 1 (2016).

[52] K.P. Katin, S.A. Shostachenko, A.I. Avkhadieva, M.M. Maslov. Adv. Phys. Chem. 2015, 1 (2015).

[53] M.M. Maslov, K.P. Katin. Chem. Phys. Lett. 644, 280 (2016).

[54] M.M. Maslov, K.P. Katin. Chem. Phys. 387, 66 (2011).

[55] И.Ю. Долинский, К.П. Катин, К.С. Гришаков, В.С. Прудковский, Н.И. Каргин, М.М. Маслов. ФТТ 60, 816 (2018).

[56] I.Y. Dolinskiy, N.V. Novikov. J. Phys. Conf. Ser. 938, 12068 (2017).

Редактор К.В. Емцев 